\title{
A NONLINEAR CONSTANT BEARING GUIDANCE AND ADAPTIVE AUTOPILOT DESIGN FOR BTT MISSILES
}

\author{
Li-Chen Fu ${ }^{1,2}$, Wei-Der Chang ${ }^{1}$, Dung-Ming Chuang ${ }^{1}$, \\ Te-Son Kuo ${ }^{1}$, Tze-Chien Wang ${ }^{1}$ and Chi-Wang Tsai ${ }^{1}$ \\ Department of Electrical Engineering ${ }^{1}$ \\ Department of Computer Science and Information Engineering ${ }^{2}$ \\ National Taiwan University, Taipei, Taiwan, R.O.C.
}

\begin{abstract}
This paper is an integraiiun of two earlier results on missile guidance and autopilot (Chuang, 1993; Chang, 1995). The guidance design is a nonlinear implementation of the constant bearing guidance. The guidance command in the form of acceleration is transformed into acceptable forms for the autopilot design. This design incorporates feedforward neural networks in the structure of adaptive control. Its weighting matrices and other unknown parameters are tuned on-line, and the approximation error of the neural networks is compensated via the technique of sliding mode control. Stability and performance for the integrated system are also investigated. 1
\end{abstract}

\section{Introduction}

Guidance processing is by itself an abundant branch of engineering. Vast different algorithms are available and are continuing to be proposed nowadays to meet different demands. Those used most widely are proportional navigation and its variants. Though they are linear in nature, it is easy to realize their underlying design concepts in nonlinear form by applying nonlinear control theory.

The bank-to-turn (BTT) policy for missile steers the missile via the motions of rolling and pitching. This control strategy arises to meet the progresses in missile airframe design such as air intake propulsive system, asymmetric geometry to increase lift force, and so on. This strategy is widely utilized for aircrafts, but not yet a mature control technology for missiles.

In this paper, we present a nonlinear guidance law design which is integrated with a neural-network based adaptive controller to realize a BTT anti-missile missile. The stability and performance of the overall system are also

\footnotetext{
1 This research is sponsored by National Science Council, R.O.C, under the grant NSC 84-2213-E-002-061.
}

investigated. The feasibility of this design is verified by simulations.

\section{System Modeling}

Equations of the system can be devided into three categories : equations of motion, kinematics and the actuator models.

Equations of Motion :

$$
\begin{aligned}
\dot{P} & =\frac{I_{y y}-I_{z z}}{I_{x x}} Q R+\frac{M_{x}}{I_{x x}} \\
\dot{Q} & =\frac{I_{z z}-I_{x x}}{I_{y y}} R P+\frac{M_{y}}{I_{y y}} \\
\dot{R} & =\frac{I_{x x}-I_{y y}}{I_{z z}} P Q+\frac{M_{z}}{I_{z z}} \\
\dot{U} & =R V-Q W+\frac{1}{m} F_{x} \\
\dot{V} & =P W-R U+\frac{1}{m} F_{y} \\
\dot{W} & =Q U-P V+\frac{1}{m} F_{z}
\end{aligned}
$$

The forces and torques in the above equations are expressed in detail as follows:

$$
\begin{aligned}
F_{x} & =C_{f_{x}} Q_{s} S_{r e f}-m g \sin \theta+T \\
F_{y} & =C_{f_{y}} Q_{s} S_{r e f}+m g \cos \theta \sin \phi \\
F_{z} & =C_{f_{z}} Q_{s} S_{r e f}+m g \cos \theta \cos \phi \\
M_{x} & =C_{m_{x}} Q_{s} S_{r e f} \bar{c} \\
M_{y} & =C_{m_{y}} Q_{s} S_{r e f} \bar{c} \\
M_{z} & =C_{m_{z}} Q_{s} S_{r e f} \bar{c} \\
\left(Q_{s}=\frac{1}{2} \rho V_{m}^{2},\right. & \text { and } \left.V_{m}^{2}=U^{2}+V^{2}+W^{2}\right)
\end{aligned}
$$

where $C_{f_{x}} \cdots C_{f_{z}}$ and $C_{m_{x}} \cdots C_{m_{z}}$ are aerodynamic coefficients with values influenced by three effective deflection angles of the tail fins, i.e. rudder deflection angle $d_{p}$, 
Kinematics :

$$
\begin{aligned}
\dot{\phi}= & P+(Q \sin \phi+R \cos \phi) \tan \theta \\
\dot{\theta}= & Q \cos \phi-R \sin \phi \\
\dot{\psi}= & (Q \sin \phi+R \cos \phi) / \cos \theta \\
{\left[\begin{array}{c}
\dot{X} \\
\dot{Y} \\
\dot{Z}
\end{array}\right]=} & {\left[\begin{array}{ccc}
c_{\psi} c_{\theta} & c_{\psi} s_{\theta} s_{\phi}-s_{\psi} c_{\phi} & c_{\psi} s_{\theta} c_{\phi}+s_{\psi} s_{\phi} \\
s_{\phi} c_{\theta} & s_{\psi} s_{\theta} s_{\phi}+c_{\psi} c_{\phi} & s_{\psi} s_{\phi} c_{\phi}-c_{\psi} s_{\phi} \\
-s_{\theta} & c_{\theta} s_{\phi} & c_{\theta} c_{\phi}
\end{array}\right] } \\
& {\left[\begin{array}{c}
U \\
V \\
W
\end{array}\right] \triangleq \mathcal{T}_{\mathcal{I B}} \cdot\left[\begin{array}{c}
U \\
V \\
W
\end{array}\right] }
\end{aligned}
$$

They describe the relation between the inertial coordinate frame and the missile body coordinate frame.

\section{Actuator Model :}

The relationships between the thrusi force and the commanded and the actual values of the effective deflection angles are modeled as single time constant low-pass filters

$$
\begin{aligned}
& \dot{d}_{p}=-\omega_{a} d_{p}+\omega_{a} u_{p} \\
& \dot{d_{q}}=-\omega_{a} d_{q}+\omega_{a} u_{q} \\
& \dot{d_{r}}=-\omega_{a} d_{r}+\omega_{a} u_{r} \\
& \dot{T}=-\omega_{a} T+\omega_{a} u_{T}
\end{aligned}
$$

where $d_{p}, d_{q}, d_{r}$ and $\dot{d}_{p}, \dot{d}_{q}, \dot{d}_{r}$ are all subject to upper and lower bounds to reflect the nonideal effects in the real world.

It is assumed that the missile accelerate with its main propulsive system after launch. Soon the fuel runs out and the missile flies mainly on its inertia. A secondary propulsive system is assumed to be equipped on the missile to maintain its speed. The thrust $T$ represents the force due to such a system.

\section{The Guidance Law Design}

The guidance algorithm here is the constant bearing guidance(Lin, 1991.) The method proposed in (Chuang, 1993) analyzes the kinematic relations between the missile and the target and derives the guidance law by nonlinear control theory.

This is done by first projecting the motions of both the missile and the target onto two planes, a horizontal plane and a vertical plane which contains both the missile center and the target center. The two planes are perpendicular to each other as depicted in Fig. 1. And the guidance design is to achieve constant bearing for the projected motions of the missile and the target on the two planes.

This relationship allows one to derive the motion of the missile and the target on the two projection planes as follows :

Horizontal plane:

$$
\dot{R}_{x y}=V_{t} \cos \gamma_{t} \cos \left(\sigma_{x y}-\chi_{t}\right)
$$

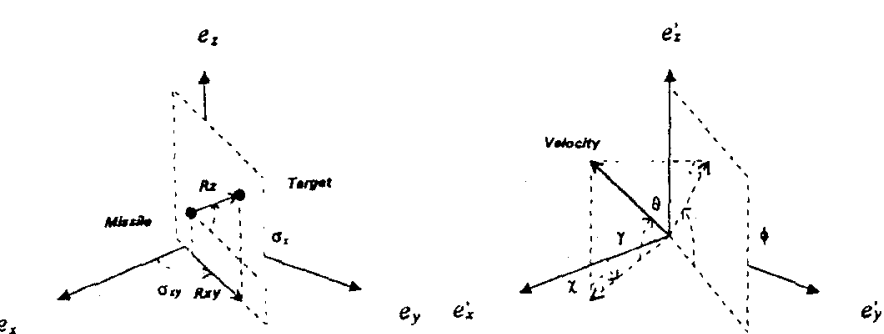

Figure 1: The projection planes and the projection of a velocity vector

$$
\begin{aligned}
R_{x y} \sigma_{x y}^{\prime}= & -V_{m} \cos \gamma_{m} \cos \left(\sigma_{x y}-\chi_{m}\right) \\
& -V_{t} \cos \gamma_{t} \sin \left(\sigma_{x y}-\chi_{t}\right) \\
& +V_{m} \cos \gamma_{m} \sin \left(\sigma_{x y}-\chi_{m}\right)
\end{aligned}
$$

Vertical plane:

$$
\begin{aligned}
\dot{R}_{z}= & V_{t} \cos \theta_{t} \cos \left(\sigma_{z}-\phi_{t}\right) \\
& -V_{m} \cos \theta_{m} \cos \left(\sigma_{z}-\phi_{m}\right) \\
R_{z} \dot{\sigma}_{z}= & -V_{t} \cos \theta_{t} \sin \left(\sigma_{z}-\phi_{t}\right) \\
& +V_{m} \cos \theta_{m} \sin \left(\sigma_{x y}-\phi_{m}\right)
\end{aligned}
$$

where $V_{t}$ and $V_{m}$ denote the magnitude of the target velocity and of the missile velocity, respectively.

In order to regulate $\dot{\sigma}_{x y}$ and $\dot{\sigma}_{z}$, the following steps are performed:

Step 1: Derive $\frac{d}{d t} \cos \theta_{t}, \frac{d}{d t} \cos \theta_{m}, \dot{\phi}_{t}$ and $\dot{\phi}_{m}$.

Step 2: With the results of step 1 , differentiate (6) and (7).The results can be denoted as $\ddot{\sigma}_{x y}$ and $\ddot{\sigma}_{z}$ as

$$
\begin{aligned}
\ddot{\sigma}_{x y} & =\frac{\Gamma_{10}}{R_{x y}}+\frac{\Gamma_{11}}{R_{x y}} \dot{\gamma}_{m}+\frac{\Gamma_{12}}{R_{x y}} \dot{\chi}_{m}+\frac{\Gamma_{13}}{R_{x y}} \dot{V}_{m} \\
\ddot{\sigma}_{z} & =\frac{\Gamma_{20}}{R_{z}}+\frac{\Gamma_{21}}{R_{z}} \dot{\gamma}_{m}+\frac{\Gamma_{22}}{R_{z}} \dot{\chi}_{m}+\frac{\Gamma_{23}}{R_{z}} \dot{V}_{m}
\end{aligned}
$$

Step 3: Design the guidance law as :

$$
\begin{aligned}
\dot{V}_{m d} & =0 \\
{\left[\begin{array}{c}
\dot{\gamma}_{m d} \\
\dot{\chi}_{m d}
\end{array}\right] } & =-\left[\begin{array}{ll}
\Gamma_{11} & \Gamma_{12} \\
\Gamma_{21} & \Gamma_{22}
\end{array}\right]^{-1}\left[\begin{array}{c}
\Gamma_{10}+a_{1} R_{x y} \dot{\sigma}_{x y} \\
\Gamma_{20}+a_{2} R_{z} \dot{\sigma}_{z}
\end{array}\right]
\end{aligned}
$$

With this design, the following are achieved :

$$
\frac{d}{d t}\left[\begin{array}{c}
\dot{\sigma}_{x y} \\
\dot{\sigma}_{z}
\end{array}\right]=\left[\begin{array}{cc}
-a_{1} & 0 \\
0 & -a_{2}
\end{array}\right]^{-1}\left[\begin{array}{c}
\dot{\sigma}_{x y} \\
\dot{\sigma}_{z}
\end{array}\right]
$$

That is, $\dot{\sigma}_{x y}$ and $\dot{\sigma}_{z}$ will approach zero exponentially.

The three guidance commands are equivalent to a threedimensional acceleration command. It is denoted as $\mathbf{a}_{m d}$ and its coordinate vector in the inertia frame is :

$$
\left[\mathbf{a}_{m d}\right]_{\text {inertia }}=\left[\begin{array}{c}
\dot{V}_{m d} \cdot \cos \gamma_{m} \cdot \cos \chi_{m} \\
\dot{V}_{m d} \cdot \cos \gamma_{m} \cdot \sin \chi_{m} \\
\dot{V}_{m d} \cdot \sin \gamma_{m}
\end{array}\right]+
$$




$$
\left[\begin{array}{c}
\dot{\gamma}_{m d} \cdot \sin \chi_{m} \\
-\dot{\gamma}_{m d} \cdot \cos \chi_{m} \\
\dot{\chi}_{m d}
\end{array}\right] \times\left[V_{m}\right]_{\text {inertia }}
$$

\section{Transformation of the Guid- ance Command}

The guidance command in the previous section is sampled and to be followed by the autopilot design in the next section. The guidance acceleration command can also be expressed in the body frame of the missile as :

$$
\left[\mathbf{a}_{m d}\right]_{b o d y}=\mathcal{I}_{\mathcal{I B}} T \cdot\left[\mathbf{a}_{m d}\right]_{\text {inertic }} \triangleq\left[\begin{array}{c}
A_{b x, d} \\
A_{b y, d} \\
A_{b z, d}
\end{array}\right]
$$

$A_{b z}$ is controlled indirectly via $\dot{W}$ as :

$$
\dot{W}_{d}=-\frac{1}{s} F(s)\left(A_{b z, d}-A_{b z}\right)
$$

where $F(s)$ is a lead compensator.

Another desired output for the autopilot is chosen as $\beta_{d}=0$, which also reders $A_{b y}=0$.

Given such, a consistent command for $A_{b y}=A_{b y, d}$ is simply $A_{b y, d}=0$, which also meets the principle of BTT.

It is done via the following procedure :

(1) Define the direction angles $\mu$ and $\sigma$ for $A_{b x, d}, A_{b y, d}, A_{b z, d}$ as

$$
\begin{aligned}
\mu & =\tan ^{-1} \frac{A_{b y, d}}{\sqrt{A_{b x, d}^{2}+A_{b z, d}^{2}}} \\
\sigma & =\tan ^{-1} \frac{A_{b x, d}}{A_{b z, d}}
\end{aligned}
$$

(2) Derive the kinematic relations :

$$
\begin{aligned}
\dot{\mu}= & P \cos \sigma-R \sin \sigma \\
\dot{\sigma}= & (P \cos \sigma-R \sin \sigma) \tan \mu \tan \sigma+Q \\
& -R \tan \mu \sec \sigma
\end{aligned}
$$

(3) The desired configuration is that $\mu_{d}=0$, which indirectly asks $A_{b y, d}=0$ when $A_{b x, d}^{2}+A_{b z, d}^{2} \neq 0$.

The final desired output for the autopilot comes directly from the guidance design as

$$
\dot{V}_{m, d}=0
$$

So far, the guidance law can be summarized by listing out the guidance commands which are transformed from the guidance acceleration command as :

$$
\begin{aligned}
\mu_{d} & =0 \\
\dot{W}_{d} & =-\frac{1}{s} F(s)\left(A_{b z, d}-A_{b z}\right) \\
\beta_{d} & =0 \\
\dot{V}_{m, d} & =0
\end{aligned}
$$

As a result of the previous explanation, the first and the third desired commands will drive $A_{b y}$ such that $A_{b y}=$ $A_{b y, d}=0$, the second one will achieve $A_{b z} \approx A_{b z, d}$, and the last desired command together with the other three will let $A_{b x} \approx A_{b x, d}$.

However, the above design is not free of defects. For example : when both of $A_{b x}$ and $A_{b z}$ are around zero, $\sigma$ may change abruptly. If the $\operatorname{sign}$ of $\sin \sigma$ or $\cos \sigma$ also changes with the abrupt change of $\sigma$, spikes in $P$ or $R$ will occur.

\section{Autopilot Design}

The autopilut design follows that of (Chang, 1996), and only the results are given in this section. The sliding surface is defined as

$$
\mathbf{S}=\left[\begin{array}{l}
\ddot{e}_{1}+2 \lambda_{1} \dot{e}_{1}+\lambda_{1}^{2} e_{1} \\
e_{2} \\
\dot{e}_{3}+\lambda_{3} e_{3} \\
e_{4}
\end{array}\right]
$$

where $e_{i}=y_{i}-y_{d i}$ and its derivative is

$$
\begin{aligned}
\dot{\mathbf{S}} & =\left[\begin{array}{c}
\mu^{(3)} \\
\ddot{W} \\
\ddot{\beta} \\
\ddot{V}_{m}
\end{array}\right]+\underbrace{\left[\begin{array}{l}
2 \lambda_{1} \ddot{e}_{1}+\lambda_{1}^{2} \dot{e}_{1} \\
-\ddot{W}_{d} \\
\lambda_{3} \dot{e}_{3} \\
0
\end{array}\right]}_{\mathbf{A}_{\mathbf{r}}} \\
& =\mathbf{A}+\mathbf{A}_{r}+\mathcal{B} \cdot \mathbf{u}+\underbrace{\left(\mathbf{A}+\mathbf{A}_{r}\right)}_{\bar{\psi}(\mathbf{x})}]+\mathcal{B} \cdot \mathbf{u} \\
& =-\Lambda \mathbf{S}+[\Lambda \mathbf{S}
\end{aligned}
$$

where $\bar{x}=\left(P, Q, R, U, V, W, d_{p}, d_{q}, d_{\tau}, \phi, \theta, \psi, \ddot{W}_{d}\right)$ and $\Lambda$ is a positive definite matrix.

As in (Chang, 1996), a feedforward neural network $\hat{\bar{\psi}}_{n}\left(\widehat{\mathcal{W}}_{\psi}, \widehat{\mathcal{V}}_{\psi}, \mathbf{x}_{a}\right)$ is built to approximate $\bar{\psi}(\mathbf{x})$, a matrix $\widehat{\Theta}_{B}$ is built to take the role of $\Theta_{B}$. The control law is designed as : Control Law:

$$
\begin{aligned}
& \mathbf{u}=\hat{B}^{-1} \cdot\left(-\Lambda \mathbf{S}-\hat{\bar{\psi}}_{n}+\mathbf{v}\right) \\
& \mathbf{v}=-\operatorname{sat}(\mathbf{S} / \epsilon) \hat{\bar{\alpha}}_{\psi}^{T} \cdot \mathbf{Y}_{\alpha}
\end{aligned}
$$

The adaptive laws are designed as : Adaptive Laws:

$$
\begin{aligned}
\dot{\widehat{\mathcal{W}}}_{\psi}^{T} & =\Gamma_{w} \cdot\left(\hat{\bar{\sigma}}--\mathcal{J}(\hat{\bar{\sigma}}) \widehat{\mathcal{V}}_{\psi} \mathbf{x}_{a}\right) \mathbf{S}_{\Delta}^{T} \\
\dot{\hat{\mathcal{V}}}_{\psi}^{T} & =\Gamma_{v} \cdot\left(\mathbf{x}_{a} \mathbf{S}_{\Delta}^{T} \widehat{\mathcal{W}}_{\psi} \mathcal{J}(\hat{\bar{\sigma}})\right) \\
\dot{\hat{\Theta}}_{B} & =\Gamma_{B} \cdot \Omega_{B} \mathbf{u} \mathbf{S}_{\Delta}^{T} \\
\dot{\overline{\hat{\alpha}}}_{\psi} & =\Gamma_{\alpha} \cdot\|\mathbf{S}\| \mathbf{Y}_{\alpha}
\end{aligned}
$$

The following two reasonable assumptions are stated here 
Assumption 1. The transformed guidance command $\dot{W}_{d}$ and its derivative $\ddot{W}_{d}$ are bounded and are within the capability of the missile.

Assumption 2. $\bar{\eta}$ is stable under the action of $\bar{\xi}$.

With these assumptions, it is proved in (Chang, 1996) that the system is stable and the tracking error will approach to a neighborhood of zero asymptotically.

\section{Closed-Loop Analysis}

When the guidance and autopilot designs are to be integrated as described in the preceeding sections, the stability and performance of the resulting integrated system have to be reaccessed. This section provides one such analysis.

For the guidance design, a Lyapunov function candidate $V_{G}$ is chosen for it as

$$
V_{G}=\frac{1}{2}\left[\begin{array}{c}
\dot{\sigma}_{x y} \\
\dot{\sigma}_{z}
\end{array}\right]^{T}\left[\begin{array}{c}
\dot{\sigma}_{x y} \\
\dot{\sigma}_{z}
\end{array}\right]
$$

And $V=V_{G}+V_{A}$ is chosen to be the Lyapunov function candidate for the integrated system.

An assumption is made to facilitate the following analysis. It is the basis of the analysis in this section.

Assumption 3 : $\left\|\mathbf{a}_{m}-\mathbf{a}_{m d}\right\|_{2} \leq b_{1}\left\|\mathbf{S}_{\Delta}\right\|_{2}+b_{2}+b_{3}(t), b_{1}$ and $b_{2}$ are positive constants, $b_{3}(t)>0$ is a fast decaying function, and $b_{2}$ is small.

From the guidance law design, it is known that

$$
\begin{aligned}
{\left[\begin{array}{c}
\dot{\sigma}_{x y} \\
\dot{\sigma}_{z}
\end{array}\right]=} & {\left[\begin{array}{cc}
\frac{1}{s+a_{1}} & 0 \\
0 & \frac{1}{s+a_{2}}
\end{array}\right] } \\
& \cdot\left[\begin{array}{ccc}
\Gamma_{13} / R_{x y} & \Gamma_{11} / R_{x y} & \Gamma_{12} / R_{x y} \\
\Gamma_{23} / R_{z} & \Gamma_{21} / R_{z} & \Gamma_{22} / R_{z}
\end{array}\right] \\
& \cdot \mathcal{T}_{A}^{-1} \cdot\left[\mathbf{a}_{m}-\mathbf{a}_{m d}\right]_{\text {inertia }}
\end{aligned}
$$

From assumption 3, if $R_{x y}, R_{z}$ are bounded away from zero, there exist $k_{1}$ and $k_{2}$ such that

$$
\begin{aligned}
& \|\left[\begin{array}{c}
\dot{\sigma}_{x y} \\
\dot{\sigma}_{z}
\end{array}\right] \cdot\left[\begin{array}{ccc}
\Gamma_{13} / R_{x y} & \Gamma_{11} / R_{x y} & \Gamma_{12} / R_{x y} \\
\Gamma_{23} / R_{z} & \Gamma_{21} / R_{z} & \Gamma_{22} / R_{z}
\end{array}\right] \\
& \cdot \mathcal{T}_{A}^{-1} \cdot\left[\mathbf{a}_{m}-\mathbf{a}_{m d}\right]_{\text {inertia }} \mid \\
& \leq\left(k_{1}\left\|\mathbf{S}_{\Delta}\right\|_{2}+k_{2}\right)\left\|\mathbf{S}_{\Delta}\right\|_{\infty}+k_{3} \\
& \leq k_{1}\left\|\mathbf{S}_{\Delta}\right\|_{\infty}^{2}+k_{2}\left\|\mathbf{S}_{\Delta}\right\|_{\infty}+k_{3}
\end{aligned}
$$

For the approach taken in this section, both the control law and adaptive laws have to be modified as followes: Modified control law :

$$
\mathbf{u}=\widehat{B}^{-1} \cdot\left(-\Lambda \mathbf{S}-\overline{\bar{\psi}}_{n}+\mathbf{v}-k_{0} \cdot \operatorname{sgn}\left(\mathbf{S}_{\Delta}\right)\right)
$$

Modified adaptive law :

$$
\begin{aligned}
\dot{\widehat{\mathcal{W}}}_{\psi}^{T} & =\Gamma_{w} \cdot\left[\left(\hat{\hat{\sigma}}-\mathcal{J}(\hat{\bar{\sigma}}) \widehat{\mathcal{V}}_{\psi} \mathbf{x}_{a}\right) \mathbf{S}_{\Delta}^{T}-\sigma \cdot \widehat{\mathcal{W}}_{\psi}^{T}\right] \\
\dot{\hat{\mathcal{V}}}_{\psi}^{T} & =\Gamma_{v} \cdot\left[\left(\mathbf{x}_{a} \mathbf{S}_{\Delta}^{T} \widehat{\mathcal{W}}_{\psi} \mathcal{J}(\hat{\bar{\sigma}})\right)-\sigma \cdot \widehat{\mathcal{V}}_{\psi}^{T}\right] \\
\dot{\widehat{\Theta}}_{B} & =\Gamma_{B} \cdot\left[\Omega_{B} \mathbf{u} \mathbf{S}_{\Delta}^{T}-\sigma \cdot \widehat{\Theta}_{B}\right] \\
\dot{\overline{\hat{\alpha}}}_{\psi} & =\Gamma_{\alpha} \cdot\left[\|\mathbf{S}\|_{2} \mathbf{Y}_{\alpha}-\sigma \cdot \widehat{\bar{\alpha}}_{\psi}\right]
\end{aligned}
$$

Stability proof :

(1) The time derivative of $V$,

$$
\begin{aligned}
& \dot{V}=-\left[\begin{array}{c}
\dot{\sigma}_{x y} \\
\dot{\sigma}_{z}
\end{array}\right]^{T}\left[\begin{array}{cc}
a_{1} & 0 \\
0 & a_{2}
\end{array}\right]\left[\begin{array}{c}
\dot{\sigma}_{x y} \\
\dot{\sigma}_{z}
\end{array}\right] \\
& +\left[\begin{array}{c}
\dot{\sigma}_{x y} \\
\dot{\sigma}_{z}
\end{array}\right]^{T}\left[\begin{array}{ccc}
\Gamma_{13} / R_{x y} & \Gamma_{11} / R_{x y} & \Gamma_{12} / R_{x y} \\
\Gamma_{23} / R_{z} & \Gamma_{21} / R_{z} & \Gamma_{22} / R_{z}
\end{array}\right] \cdot \\
& \mathcal{T}_{A}^{-1} \cdot\left[\mathbf{a}_{m}-\mathbf{a}_{m d}\right]_{\text {inertia }}-\mathbf{S}_{\Delta}^{T} \Lambda \mathbf{S}_{\Delta}-\frac{\sigma}{2} t r\left\{\widetilde{\mathcal{W}}_{\psi} \widehat{\mathcal{W}}_{\psi}^{T}\right\} \\
& -\frac{\sigma}{2}\left(\operatorname{tr}\left\{\widetilde{\mathcal{V}}_{\psi} \widehat{\mathcal{V}}_{\psi}^{T}\right\}-\operatorname{tr}\left\{\widetilde{\Theta}_{B}^{T} \widehat{\Theta}_{B}\right\}-\operatorname{tr}\left\{\widetilde{\tilde{\alpha}}_{\psi}^{T} \widehat{\bar{\alpha}}_{\psi}\right\}\right)-k_{0}\left\|\mathbf{S}_{\Delta}\right\|
\end{aligned}
$$

(2) Denote $\theta^{*}$ as true value of all the parameters, and $\tilde{\theta}$ as the estimation error,

$$
\begin{aligned}
\dot{V} \leq & -\bar{k}_{0} V+k_{1}\left\|\left(\mathbf{S}_{\Delta}\right)_{t}\right\|_{\infty}^{2}+k_{2}\left\|\left(\mathbf{S}_{\Delta}\right)_{t}\right\|_{t, \infty} \\
& +\sigma\|\tilde{\theta}\|\left\|\theta_{*}\right\|+k_{3}
\end{aligned}
$$

(3) Let $V_{\infty}(t)=\left\|(V(t))_{t_{1}}\right\|_{\infty}$

$$
\dot{V}+\bar{k}_{0} V \leq \bar{k}_{1} V_{\infty}+\bar{k}_{2} V_{\infty}^{\frac{1}{2}}+\bar{k}_{3}
$$

(4) From this, it is solved that

$$
\begin{array}{r}
V(t) \leq \int_{0}^{t} e^{-\bar{k}_{0}\left(t-t^{\prime}\right)}\left(\bar{k}_{1} V_{\infty}+\bar{k}_{2} V_{\infty}^{1 / 2}+\bar{k}_{3}\right) d t^{\prime}+c e^{-\bar{k}_{0} t} \\
\Rightarrow V_{\infty}(t) \leq \frac{1}{\bar{k}_{0}}\left(\bar{k}_{1} V_{\infty}+\bar{k}_{2} V_{\infty}^{1 / 2}+\bar{k}_{3}\right)+c
\end{array}
$$

(5) If $k_{0}$ satisfies $1-\frac{\bar{k}_{1}}{\bar{k}_{0}}>0$, then $\exists \kappa>0$ such that

$$
0 \leq V_{\infty}(t) \leq \kappa
$$

Also from assumption 2, it is proved that the closed loop system is stable.

Performance analysis :

A theorem from (LaSalle, 1961) is included here :

Theorem (LaSalle, 1961)

For the system $\dot{x}=f(x)$. Let $V(x)$ be a radially unbounded $C^{1}$ scalar function If $\dot{V}(x) \leq-\varepsilon<0$ for all $x$ outside some closed and bounded set $M$, then $x$ is ultimately bounded.

(1) Boundedness of $\theta^{*}$ and $\tilde{\theta}$ imply that if the adaptive gains are chosen to be very alrge

$$
\begin{aligned}
V_{A} & =\frac{1}{2} \mathbf{S}_{\Delta}^{T} \mathbf{S}_{\Delta}+\sigma_{1} \\
\dot{V}_{A} & =-\mathbf{S}_{\Delta}^{T} \Lambda \mathbf{S}_{\Delta}-k_{0}\left\|\mathbf{S}_{\Delta}\right\|_{2}+\sigma_{2} \\
& \leq-\bar{k}_{0}\left\|\mathbf{S}_{\Delta}\right\|_{2}^{2}-k_{0}\left\|\mathbf{S}_{\Delta}\right\|_{2}+\sigma_{2} \\
& =-\bar{k}_{0}\left(\left\|\mathbf{S}_{\Delta}\right\|-\delta_{1}\right)\left(\left\|\mathbf{S}_{\Delta}\right\|+\delta_{2}\right)
\end{aligned}
$$

where $\delta_{1}, \delta_{2}>0, \sigma_{1}$ and $\sigma_{2}$ are bounded functions. Moreover, $\sigma_{1}$ is very small when $\sigma$ is very large. 


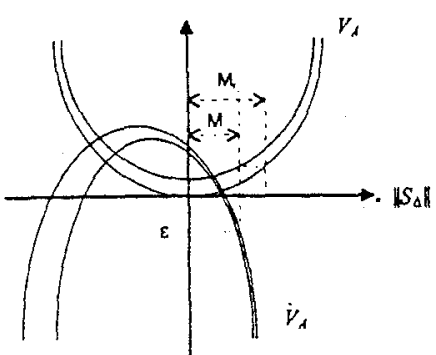

Figure 2: The relation between $M$ and the ultimate bound $M_{r}$

(2) If $k_{0} \gg 0$, then $\left\|\mathbf{S}_{\Delta}\right\|_{2}>\delta_{1} \approx \frac{2 \bar{k}_{0}}{k_{0}} \Rightarrow \dot{V}_{A} \leq$ 0 . By the theorem above, it is known that $\left\|\mathbf{S}_{\Delta}\right\|$ is ultimately bounded by $M_{r}$ (in the order of $\frac{2 \bar{k}_{0}}{\dot{k}_{0}}$ ) as in Fig. 2, as is indicated in (LaSalle, 1961).

(3) From assumption 3, it follows that $\left\|\mathbf{a}_{m}-\mathbf{a}_{m d}\right\|$ approaches to a neighborhood of zero.

\section{Computer Simulation}

When the miss distance is within $100(\mathrm{~m})$, the guidance command is ignored. The simulation is stopped when the miss distance is within $5(\mathrm{~m})$. The modifications on the control and adaptive laws are not applied in the simulations.

\begin{tabular}{|l||r|r|r|}
\hline Target & $\mathrm{X}$ & $\mathrm{Y}$ & $\mathrm{Z}$ \\
\hline \hline Initial Position $(\mathrm{m})$ & 6000.0 & 8000.0 & 15000.0 \\
\hline Initial Velocity $(\mathrm{m} / \mathrm{s})$ & -1000.0 & -1500.0 & -1800.0 \\
\hline Acceleration $\left(\mathrm{m} / \mathrm{s}^{2}\right)$ & $-0.1 V_{y}$ & $0.1 \mathrm{~V}_{z}$ & -9.8 \\
\hline
\end{tabular}

\begin{tabular}{|l||r|r|r|}
\hline Missile & $\mathrm{X}$ & $\mathrm{Y}$ & $\mathrm{Z}$ \\
\hline Initial Position $(\mathrm{m})$ & 0.0 & 0.0 & 3000.0 \\
\hline \hline & $\phi$ & $\theta$ & $\psi$ \\
\hline Euler Angle $(\mathrm{rad})$ & $\pi$ & $-\frac{1}{3} \pi$ & $-\frac{1}{4} \pi$ \\
\hline \hline & $U$ & $V$ & $W$ \\
\hline Initial Velocity $(\mathrm{m} / \mathrm{s})$ & 1000.0 & 0.0 & 150.0 \\
\hline
\end{tabular}

\section{Conclusions}

To be more specific, the main features of the design in this thesis include: it can be applied to intercept invading, fast-moving, and maneuvering targets; the stability of the integrated system is also investigated.

However, such a design is not free of defects : there exist unavoidable singular conditions when the guidance design fails to work properly; the autopilot design cannot handle small but fast time-varying acceleration command very well; the acceleration of the missile is not controlled with high precision.

\section{REFERENCES}

Chuang , D.-M., "Guidance and Nonlinear Control for A Highly Maneuvable Missile," Master's Thesis, National Taiwan University, 1993.

Chang, W.-D., "Adaptive Robust Neural Network-Based Control for Bank-to-Turn Missiles," Preprint of the 13th World Congress of IFAC, vol. P, pp. $237-242$.

LaSalle, J.P. and Lefschetz, Stability by Lyapunov's Direct Method : with Applicatio ns, Academic Press, 1961.

Lin , C.F., Modern Navigation Guidance, and Control Processing, Prentice-Hall, 1991.
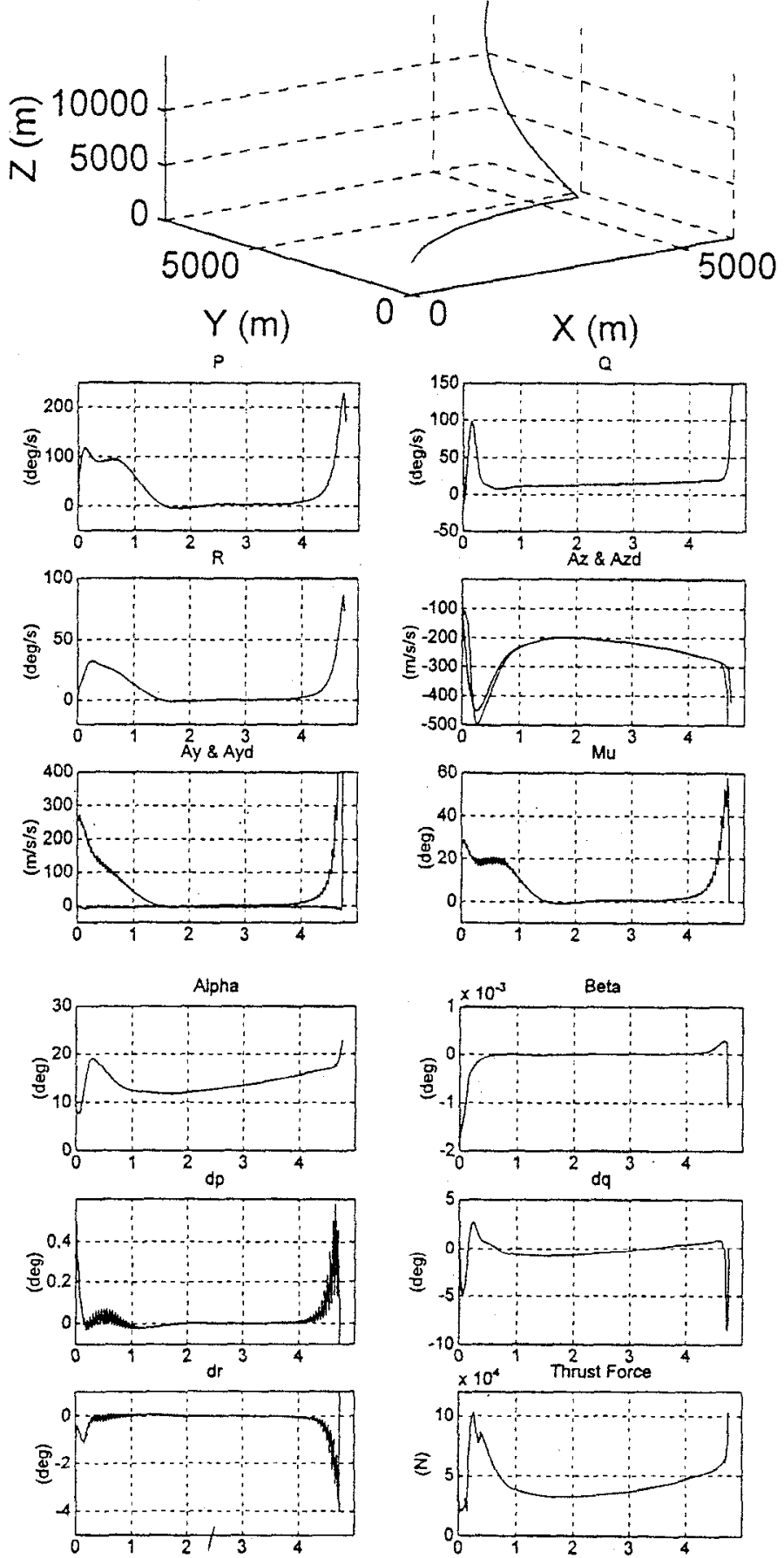